\title{
Construcciones geométricas en GeoGebra a partir de diferentes sistemas de representación: un estudio con maestros de primaria en formación
}

\author{
Geometric constructions in GeoGebra from different \\ representation systems: a study with prospective primary \\ education teachers
}

\author{
Alberto Arnal-Bailera ${ }^{1}$ \\ Antonio M. Oller-Marcén²
}

\begin{abstract}
Resumen: En este trabajo estudiamos la realización con GeoGebra de tres procedimientos de construcción hechos a partir de las instrucciones presentadas en dos ediciones diferentes de los Elementos de Euclides: la clásica y la menos conocida de Oliver Byrne. Estas ediciones emplean sistemas de representación radicalmente diferentes para presentar un mismo contenido matemático. Analizamos la influencia de estos sistemas de representación en la producción de construcciones correctas y en el seguimiento de las instrucciones planteadas, en las respuestas de 18 parejas de alumnos de tercer curso del Grado de Magisterio de Educación Primaria. Encontramos que el sistema de representación no influye sobre el seguimiento fiel de las instrucciones, Pero, en el caso de la producción de construcciones correctas, la influencia del sistema de representación está asociada a la tarea concreta. Identificamos y analizamos cuatro fenómenos relacionados con aspectos matemáticos de instrumentación y de instrumentalización.
\end{abstract}

Palabras clave: Construcción geométrica, GeoGebra, Sistemas de representación, Formación de profesores.

Fecha de recepción: 6 de febrero de 2019. Fecha de aceptación: 12 de noviembre de 2019 .

${ }^{1}$ Facultad de Educación, Universidad de Zaragoza. albarnal@unizar.es, orcid.org/0000-0002-0516-0463.

2 Centro Universitario de la Defensa de Zaragoza. oller@unizar.es, orcid.org/0000-0002-8191-3199. 


\begin{abstract}
In this work, we study the implementation using GeoGebra of three construction processes as described in two different editions of Euclid's Elements: the classical and the less known one by Oliver Byrne. These editions use dramatically different representation systems to present the same mathematical content. We analyze the influence between the representation systems, the correctness of the constructions and the adjustment to the given instructions shown in the answers of 18 pairs of third year students of the Degree of Primary Education. We observe no influence of the representation systems over the adjustment while in the case of correctness this influence is associated to the particular task. In addition, we identify and analyze four different phenomena related to mathematical, instrumentation and instrumentalization aspects.
\end{abstract}

Keywords: Geometric construction, GeoGebra, Representation systems, Teacher training.

\title{
1. INTRODUCCIÓN Y OBJETIVOS
}

Múltiples ejercicios de geometría clásica consisten en hacer construcciones geométricas a partir de algunos de los elementos que las constituyen (construcción de un polígono regular dado uno de sus lados, construcción de un triángulo a partir de sus tres lados, etc.) o de representaciones de objetos que cumplan ciertas propiedades (construcción de una recta perpendicular a otra por un punto, construcción de una recta tangente a una circunferencia por un punto exterior, etc.). Estas construcciones se han realizado tradicionalmente con regla y compás siguiendo procedimientos más o menos estandarizados. Textos clásicos, como los Elementos, incluyen un amplio catálogo de tales construcciones detallando paso a paso las acciones a realizar con los citados instrumentos. El uso de un software de geometría dinámica (de GeoGebra en particular) para llevar a cabo este tipo de construcciones implica, por sus especificidades, una reinterpretación o adaptación de los pasos descritos en los textos clásicos, que va más allá de la mera traslación del procedimiento.

Esta adaptación, no obstante, no está exenta de dificultades por cuanto que las instrucciones proporcionadas en los textos están concebidas para ser seguidas utilizando herramientas concretas (la regla y el compás) y no programas informáticos. La labor de adaptación debe ser hecha por los docentes a la hora 
de diseñar y preparar sus clases o de generar recursos o actividades para sus alumnos, en relación con estos contenidos.

En este sentido, una tarea propia de la formación del profesorado en didáctica de la geometría, es la realización de construcciones con regla y compás, en ocasiones con la ayuda de GeoGebra, con el objetivo de analizar, o de ser conscientes al menos, de los elementos matemáticos que sustentan las mismas. Una de las dificultades que encuentran los maestros en formación al llevar a cabo este tipo de tareas está relacionada con la correcta interpretación de las instrucciones proporcionadas para llevar a cabo la construcción (Özerem, 2012). Surge, por tanto, la siguiente pregunta de investigación: ¿̇La utilización de un sistema de representación particular en las instrucciones proporcionadas a los alumnos en un procedimiento de construcción geométrica, mejora sus resultados cuando la realizan usando el software GeoGebra? Para tratar de responder a esta pregunta abordamos los siguientes objetivos específicos:

1. Determinar si existe interdependencia entre el sistema de representación usado para proporcionar las instrucciones a los alumnos, el seguimiento de las instrucciones y la corrección de las construcciones realizadas en GeoGebra.

2. Analizar los procedimientos de construcción llevados a cabo por los alumnos al realizar las construcciones que se les solicitan, en relación con los distintos sistemas de representación que usan y con los procesos de instrumentalización e instrumentación que adelantan.

\section{MARCO TEÓRICO}

Duval (2006, p. 107) señala que "ningún proceso matemático puede ser llevado a cabo sin utilizar un sistema de representación semiótico puesto que los procesos matemáticos siempre implican la sustitución de una representación semiótica por otra". De hecho, Gómez (2002) considera el estudio de los sistemas de representación como uno de los pilares básicos del análisis de contenido considerado dentro del análisis didáctico definido por Rico (2013).

Desde el punto de vista de la enseñanza de las matemáticas, lo anterior implica que los estudiantes deberían ser capaces de reconocer un mismo objeto matemático con independencia de dicho contexto (Duval, 2004). En particular, tal y como señalan Castro y Castro (1997, p. 103): "Dominar un concepto matemático consiste en conocer sus principales representaciones y el significado de 
cada una de ellas, así como operar con las reglas internas de cada sistema y en convertir o traducir unas representaciones en otras, detectando qué sistema es más ventajoso para trabajar con determinadas propiedades".

Esta capacidad de moverse entre distintos sistemas de representación se denomina en ocasiones fluidez multi-representacional y existen evidencias de que puede ser promovida con el apoyo de entornos tecnológicos y, en particular, de GeoGebra (McGee y Moore-Russo, 2015).

En el caso de la geometría, parece claro que el registro o representación figural juega un papel fundamental en el aprendizaje, relacionado con aspectos tales como la visualización o la abducción (Marmolejo y Vega, 2012). A este respecto, al considerar las ilustraciones que a menudo acompañan a las construcciones geométricas, hemos de prestar atención a las diferencias entre una figura, un objeto teórico que sirve como referente (definido por sus propiedades matemáticas) y un dibujo, una representación particular en la pantalla o el papel de dicho objeto abstracto (Laborde y Capponi, 1994). Un dibujo, de hecho, contendrá particularidades y casi siempre inexactitudes pues es inevitable una cierta pérdida de información con respecto al objeto geométrico que representa (Parzysz, 1988). Pese a ello, existen estudios que revelan una tendencia por parte de los alumnos a llevar a cabo razonamientos basados más en su percepción de un dibujo que en la información que se les proporciona (Sandoval, 2009).

En consecuencia, al encontrarnos con una ilustración que acompaña a una construcción o problema geométrico, pueden darse dos situaciones (Mesquita, 1998). Por una parte, si esta ilustración es vista como una figura, sus propiedades pueden utilizarse en los razonamientos. Sin embargo, cuando se trata de un dibujo no siempre se puede saber qué objeto teórico representa y no es fácil extraer información de ésta. Para poder hacerlo, si se utiliza lápiz y papel se debería incorporar el uso de algún tipo de convención sobre el dibujo que permita añadir información adicional; mientras que, si se utiliza GeoGebra, sería necesario conocer las herramientas usadas para su construcción y las propiedades matemáticas derivadas de las mismas (Gutiérrez, 2005).

Como ya hemos apuntado, el uso de GeoGebra como artefacto didáctico (Rezat y Sträßer, 2012) puede facilitar el trabajo con distintos sistemas de representación. Desde un punto de vista general, la utilización de artefactos en el aula, entendidos como cualquier elemento susceptible de ser usando intencionalmente en actividades de enseñanza y aprendizaje, conlleva la aparición de un fenómeno denominado génesis instrumental (Rabardel, 1995; Trouche, 2004). La génesis instrumental es un proceso personal de cada 
individuo participante en una situación de enseñanza y aprendizaje que involucra en cierto modo la transformación de un artefacto en un instrumento. Así, el artefacto, que es exclusivamente un objeto físico, se transforma en algo más complejo que incluye, además de lo físico, una parte cognitiva que aporta cada individuo y que le confiere una funcionalidad que le permite integrarlo en su actividad. Esto se produce gracias a la emergencia conjunta de esquemas mentales y técnicas de uso de la herramienta cuando se trabaja, por ejemplo, con tecnologías digitales (Drijvers, 2012).

En el proceso de génesis instrumental, Rabardel (1995) distingue dos subprocesos, uno orientado al artefacto y otro al individuo. En el primero, la instrumentalización, el individuo influye en el artefacto, descubriendo sus funcionalidades personalizando sus utilidades o utilizándolo para fines no previstos por el diseñador. En el segundo subproceso, la instrumentación, el artefacto influye en el individuo permitiéndole desarrollar una cierta actividad matemática de un modo distinto a como posiblemente venía realizándola. Este proceso de génesis instrumental es guiado por el docente mediante la organización sistemática e intencional de un entorno de aprendizaje que Trouche (2004) denomina orquestación instrumental.

Diversos autores se han dedicado a analizar estos procesos de génesis instrumental en un contexto tecnológico. Guin y Trouche (2002), por ejemplo, identifican distintos comportamientos de los estudiantes al enfrentarse al manejo del instrumento (comportamiento del pescador, transporte automático o determinación inflexible) en función de la complejidad de su interacción con el instrumento y del grado de reflexión del alumno. En un contexto de geometría dinámica y trabajando con futuros maestros de primaria, Ruíz-López (2018) identifica algunos factores que pueden dificultar una completa génesis instrumental. En particular, señala posibles deficiencias en el conocimiento matemático de los estudiantes, un desarrollo limitado de la instrumentalización en el uso de GeoGebra o un imperfecto proceso de orquestación instrumental.

En muchas ocasiones el uso de GeoGebra se combina o complementa, sin sustituir totalmente, a los instrumentos tradicionales. En estas situaciones se debe tener en cuenta la distinta relevancia del orden en que se realizan los pasos de una construcción y su interdependencia. Talmon y Yerushalmy (2004) afirman que esta relevancia es mayor en los entornos tecnológicos que en los tradicionales.

Iranzo y Fortuny (2009) detallan tareas que muestran la dificultad de transferencia de las estrategias de resolución con papel y lápiz a las estrategias con ayuda de GeoGebra y viceversa, estableciendo tipologías de alumnos según la 
mayor o menor presencia de prácticas de reflexión o procedimentales: autónomos (alto nivel de instrumentación e instrumentalización), instrumentales (nivel medio de instrumentación e instrumentalización), procedimentales (nivel bajo de instrumentalización) y naif, (nivel bajo de instrumentación). En aquellas situaciones en las que se hace un uso simultáneo del software y del lápiz y papel, Iranzo y Fortuny (2009) también señalan que alumnos con un grado de instrumentación bajo (tipo naïf) en ocasiones recurren a herramientas relacionadas con la medida al utilizar GeoGebra incluso en situaciones en las que esto no es necesario. Además, en estos casos los alumnos pueden presentar también dificultades conceptuales, algebraicas y de visualización, utilizando GeoGebra como si fuera una pizarra tradicional (Arnal-Bailera y Oller-Marcén, 2017).

Un interesante indicador del grado de instrumentación alcanzado por los estudiantes en el uso de GeoGebra está relacionado con los tipos de arrastres que estos realizan al abordar una construcción geométrica. Arzarello et al. (2002) desarrollan una taxonomía en la que incluyen hasta siete tipos distintos: arrastre errante, arrastre unido, arrastre guiado, arrastre lugar oculto, arrastre de línea, arrastre enlazado y test de arrastre. Los que más nos interesan aquí, son el arrastre guiado (mover puntos de un objeto para darle una forma determinada), el arrastre lugar oculto (mover puntos de un objeto para que este siga manteniendo ciertas propiedades) y el test de arrastre (mover puntos para determinar si el objeto al que pertenece mantiene las propiedades deseadas).

Generalmente, las construcciones llevadas a cabo por alumnos con un bajo nivel de instrumentación no pasan el test de arrastre (Mariotti y Bartolini, 1998). De hecho, utilizando la distinción descrita anteriormente, esto es equivalente a decir que estos alumnos tienden a construir un dibujo y no una figura. De hecho, un arrastre adecuado de los puntos libres de una construcción permite saber qué propiedades tiene una representación y por lo tanto qué figura representa; es decir, se puede saber si la representación lograda es la deseada o no y si se realizó el procedimiento de manera correcta o incorrecta. Este es un asunto normativo (cláusula del contrato didáctico) de la clase en lo que respecta a la corrección o no de un procedimiento de construcción al que hacen referencia Laborde y Capponi (1994).

\section{MÉTODO Y PARTICIPANTES}

El estudio realizado es de carácter exploratorio y tiene una finalidad esencialmente descriptiva (Elliot y Timulak, 2005). Se trata de una investigación de tipo mixto en la 
que se combina el enfoque cuantitativo con el análisis de datos de tipo cualitativo (Hernández, Fernández y Baptista, 2010). El uso de metodologías de tipo mixto permite que los enfoques cuantitativo y cualitativo "puedan complementarse para explorar distintos aspectos de la misma pregunta" (Pole, 2009, p. 39). En nuestro caso, el primero de los objetivos es de carácter descriptivo y pretende determinar la existencia de relación entre variables de tipo categórico (Blaikie, 2003) por lo que la metodología utilizada es de carácter cuantitativo. El segundo objetivo tiene un carácter más interpretativo y la metodología adoptada es cualitativa. En este sentido, nuestro estudio presenta los rasgos que Teddlie y Tashakkori (2009) señalan como característicos de las metodologías mixtas de investigación: utilización de datos tanto numéricos como narrativos, integración de análisis estadísticos y temáticos, combinación de propósitos confirmatorios y exploratorios, etc.

La experimentación se llevó a cabo con 36 estudiantes del Grado en Magisterio de Educación Primaria durante el curso académico 2014-2015, en el marco de la asignatura Didáctica de la Geometría que se desarrolla en el segundo cuatrimestre del tercer curso. En concreto, la actividad se desarrolló en una sesión de dos horas de duración en el aula de informática. Durante la sesión se combinó el trabajo de los alumnos en 18 pequeños grupos formados por hasta tres personas, con las puestas en común coordinadas por los investigadores.

El cuestionario sobre el que trabajaron los alumnos constaba de tres tareas. Cada una de ellas consistía en la realización de una construcción geométrica. La Tarea 1 consistía en la construcción de un cuadrado a partir de un lado (Proposición 46 del Libro I de los Elementos), la Tarea 2 en la construcción de una tangente a una circunferencia por un punto exterior a ella (Proposición 17 del Libro III de los Elementos) y la Tarea 3 en la construcción de un triángulo a partir de tres segmentos tales que la suma de cada dos de ellos es mayor que el tercero (Proposición 22 del Libro I de los Elementos). En concreto, los alumnos debían reproducir las instrucciones proporcionadas por escrito, mediante el uso de GeoGebra, indicando, tal y como se observa en las Figuras 1 a 3, las herramientas utilizadas para realizar cada uno de los pasos de la construcción. Además, para cada una de las tres tareas, los alumnos disponían de un fichero GeoGebra en el que se proporcionaban los elementos iniciales sobre los que se debía realizar la construcción.

Para alcanzar los objetivos planteados anteriormente, se crearon dos versiones del cuestionario. En una de ellas (que llamaremos versión C), las instrucciones y figuras proporcionadas a los alumnos provenían de la edición clásica de los Elementos de Euclides (Euclides, trad. en 1994). En la otra (que 
llamaremos versión B), se proporcionaron las instrucciones y figuras tal y como aparecen en la edición de los Elementos realizada por el inglés Oliver Byrne (Byrne, 1847). De los 18 grupos, 10 completaron el modelo B del cuestionario, mientras los 8 restantes completaron el modelo C. En la versión C se evitó el uso de letras del alfabeto griego, que fueron sustituidas por letras mayúsculas del alfabeto latino.

En las Figuras 1 a 3 se muestran las dos versiones de cada una de las tareas que conforman el cuestionario. En ellas se pueden apreciar los distintos sistemas de representación utilizados. La edición de Oliver Byrne de los Elementos de Euclides (mitad superior de las Figuras 1, 2 y 3) utiliza un sistema de representación poco convencional. El sistema tradicional (letras mayúsculas para puntos, segmentos denotados a partir de sus extremos, etc.), que se puede apreciar en la edición clásica (mitad inferior de las Figuras 1, 2 y 3), es sustituido por un lenguaje de tipo fundamentalmente icónico en el que el color y la representación intuitiva de objetos y conceptos resulta de gran importancia. El uso del sistema de representación planteado por Byrne se ha utilizado recientemente de forma fructífera en trabajos con profesorado de secundaria en formación (Arnal-Bailera y Oller-Marcén, 2017). En la Tabla 1 se presentan las principales características y diferencias entre ambos sistemas de representación. Las Figuras 1 a 3 ponen de manifiesto de forma ostensiva las particularidades, ventajas e inconvenientes de cada uno de ellos.

Tabla 1. Características principales de los sistemas de representación considerados.

\begin{tabular}{|l|l|l|}
\cline { 2 - 3 } \multicolumn{1}{l|}{} & \multicolumn{1}{c|}{$\begin{array}{c}\text { Versión C } \\
\text { (Euclides, 1994) }\end{array}$} & \multicolumn{1}{c|}{$\begin{array}{c}\text { Versión B } \\
\text { (Byrne, 1847) }\end{array}$} \\
\hline Color & No & $\begin{array}{l}\text { Sí. A cuatro colores (azul, ne- } \\
\text { gro, amarillo y rojo) }\end{array}$ \\
\hline Trazos y grosores & Único & $\begin{array}{l}\text { Distintos grosores y trazos } \\
\text { (continuo, punteado, etc.) }\end{array}$ \\
\hline $\begin{array}{l}\text { Referencia a objetos geométri- } \\
\text { cos }\end{array}$ & Mediante letras & Mediante iconos \\
\hline $\begin{array}{l}\text { Distinción entre objetos geomé- } \\
\text { tricos diferentes }\end{array}$ & Distintas letras & $\begin{array}{l}\text { Distintos colores, grosores, tra- } \\
\text { zos, etc. }\end{array}$ \\
\hline $\begin{array}{l}\text { Expresión de relaciones entre } \\
\text { objetos geométricos }\end{array}$ & $\begin{array}{l}\text { Lenguaje verbal especializado } \\
\text { (perpendicular, paralela, etc.) }\end{array}$ & $\begin{array}{l}\text { Lenguaje simbólico especiali- } \\
\text { zado ( } \perp \text {, ll, etc.) }\end{array}$ \\
\hline
\end{tabular}


Como se observa en la Figura 1, en ambos casos el procedimiento general de construcción del cuadrado es similar. La principal diferencia radica en que la versión de Byrne unifica, en cierto modo, los dos pasos iniciales, proponiendo dibujar un segmento perpendicular al segmento dado y de su misma longitud. La versión clásica propone inicialmente el trazado de un segmento perpendicular al dado, sobre el que posteriormente se traslada la longitud de éste. También se observa que en el paso final la versión de Byrne proporciona información redundante al señalar que los dos últimos segmentos dibujados sean concurrentes.

En la Tarea 2 se aprecian mayores diferencias entre ambos procedimientos. El primer paso es el mismo en ambos casos; sin embargo, los dos siguientes están invertidos. En la versión de Byrne se comienza dibujando una perpendicular al segmento dibujado en el primer caso y a continuación se traza una circunferencia, mientras que en la versión clásica se construye primero la circunferencia. También resulta interesante señalar que en la versión de Byrne se omite la indicación explícita del trazado de las dos últimas líneas auxiliares de la construcción que, sin embargo, sí aparecen en la figura.

La Tarea 3 es la que presenta aparentemente mayores diferencias entre ambas versiones. La versión clásica implica la traslación de los tres segmentos dados sobre una misma semirrecta, mientras que en la versión de Byrne basta con trasladar dos de los segmentos usando como punto común en cada caso uno de los extremos del otro sin necesidad de que estén alineados. Aunque no tiene impacto sobre el proceso de construcción, es interesante señalar que la desigualdad triangular (la suma de dos cualesquiera de los segmentos debe ser mayor que el tercero) recibe un tratamiento ligeramente distinto en ambos textos. Mientras que en la versión de Byrne aparece como hipótesis, en la versión clásica se resalta la necesidad de este hecho.

La recogida de la información se llevó a cabo mediante diversos procedimientos. El primero consistió en la observación por parte de los investigadores. En segundo lugar, se dispuso de las producciones de los alumnos tanto escritas, en sus respuestas al cuestionario, como digitales en los ficheros de GeoGebra generados. 
TAREA 1: Sigue las instrucciones para construir un cuadrado de lado dado en GeoGebra a partir de la siguiente proposición. Anota las herramientas de GeoGebra que vayas utilizando.

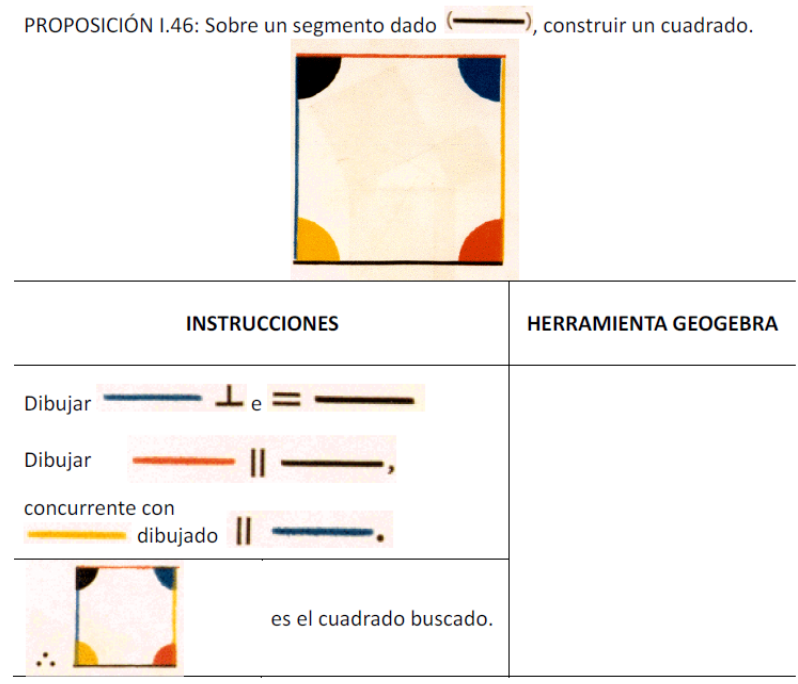

TAREA 1: Sigue las instrucciones para construir un cuadrado de lado dado en GeoGebra a partir de la siguiente proposición. Anota las herramientas de GeoGebra que vayas utilizando.

PROPOSICIÓN I.46: Trazar un cuadrado a partir de una recta dada.

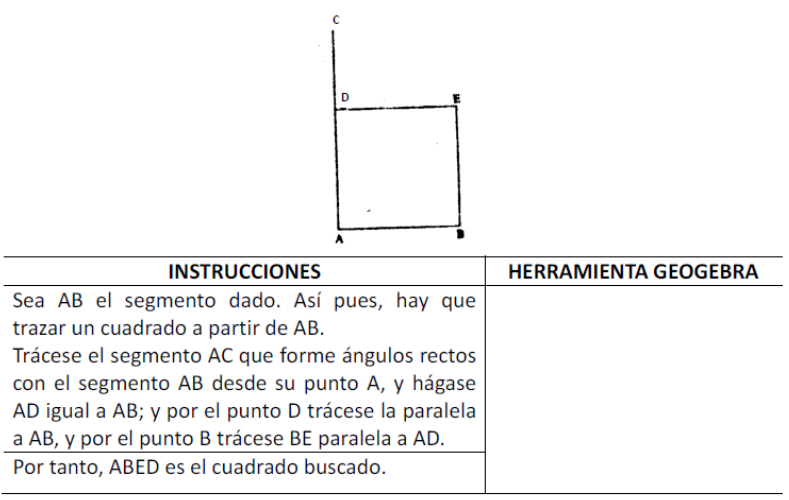

Figura 1. Enunciado de la Tarea 1 en ambas versiones del cuestionario. 
TAREA 2: Construye una tangente a una circunferencia por un punto exterior en GeoGebra a partir de la siguiente proposición. Anota las herramientas de GeoGebra que vayas utilizando.

PROPOSICION III.17: Para dibujar una tangente a un círculo dada desde un punto dado, exterior a la circunferencia.
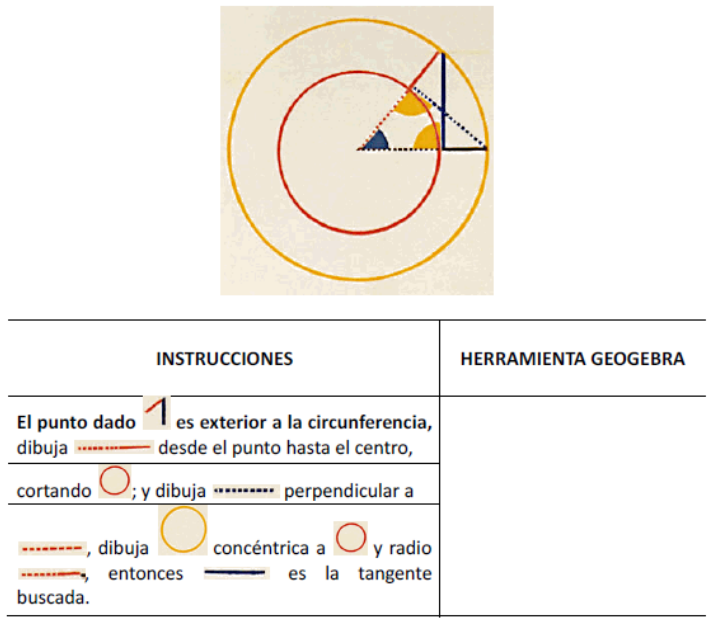

TAREA 2: Construye una tangente a una circunferencia por un punto exterior en GeoGebra a partir de la siguiente proposición. Anota las herramientas de GeoGebra que vayas utilizando.

PROPOSICION III.17: Desde un punto dado trazar una línea tangente a un círculo dado.

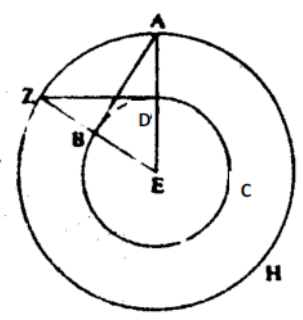

\begin{tabular}{l|l}
\hline \multicolumn{1}{c|}{ INSTRUCCIONES } & HERRAMIENTA GEOGEBRA \\
\hline Sea el punto dado $A$ y la circunferencia dada $B C D$. & \\
\cline { 1 - 1 } Tómese, pues, el centro de la circunferencia, $E, y$ & \\
trácese $A E$ y con el centro $E$ y la distancia $E A$ & \\
descríbase la circunferencia $A Z H, y$ a partir del & \\
punto $D$ trácese $D Z$ formando ángulos rectos con & \\
$E A, y$ trácense EZ, AB. & \\
\cline { 1 - 1 } Digo que ha sido trazada $A B$ desde el punto $A$ & \\
tangente a la circunferencia $B C D$. &
\end{tabular}

Figura 2. Enunciado de la Tarea 2 en ambas versiones del cuestionario. 
TAREA 3 Construye un triángulo en GeoGebra a partir de la siguiente proposición. Anota las herramientas de GeoGebra que vayas utilizando.

PROPOSICION 1.22: Dados tres segmentos - en los que la suma de dos cualesquiera de ellos es mayor que el tercero, construir un triángulo cuyos lados deben ser iguales respectivamente a los segmentos dados.
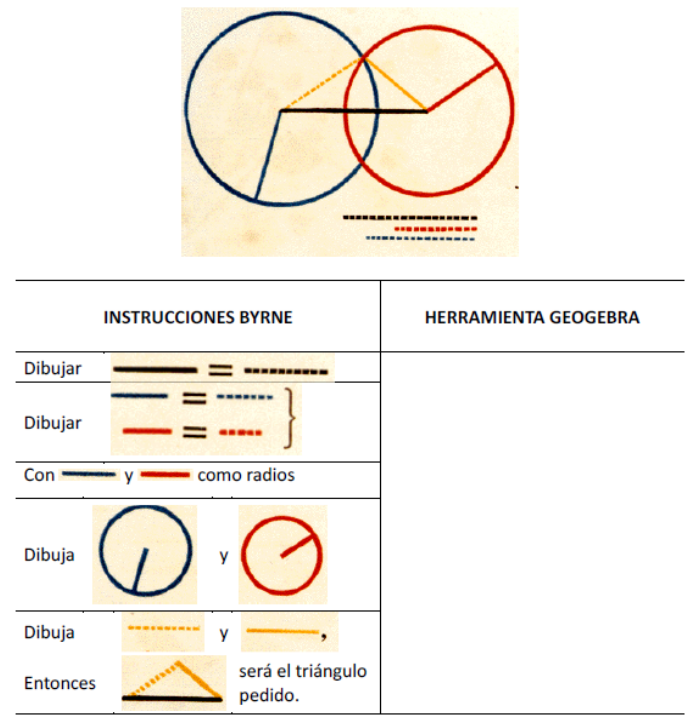

TAREA 3 Construye un triángulo en GeoGebra a partir de la siguiente proposición. Anota las herramientas de GeoGebra que vayas utilizando.

PROPOSICION I.22: Construir un triángulo con tres segmentos que son iguales a tres segmentos dados. Pero es necesario que dos de los segmentos tomados juntos de cualquier manera sean mayores que el restante.

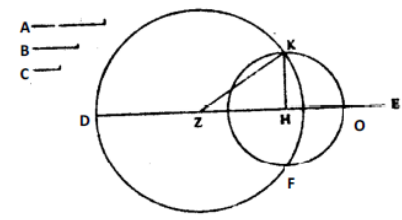

\begin{tabular}{|c|c|}
\hline INSTRUCCIONES & HERRAMIENTA GEOGEBRA \\
\hline $\begin{array}{l}\text { Sean A, B, C los tres segmentos dados, } \\
\text { cumpliendo las condiciones del } \\
\text { enunciado. }\end{array}$ & \\
\hline $\begin{array}{l}\text { Póngase una semirrecta que parte de } \\
D, y \text { hágase } D Z \text { igual a } A \text {, }\end{array}$ & \\
\hline y $\mathrm{ZH}$ igual a $\mathrm{B}$ y $\mathrm{HO}$ igual a C; & \\
\hline $\begin{array}{l}\text { y con el centro Z y la distancia ZD } \\
\text { descríbase la circunferencia DKF; }\end{array}$ & \\
\hline $\begin{array}{lll}\text { Así mismo, con el centro } \mathrm{H} \text { y la } \\
\text { distancia } \mathrm{HO} \text { descríbase la } \\
\text { circunferencia } \mathrm{KFO} \text {, y trácense } \mathrm{KZ}, \mathrm{KH} \text {. }\end{array}$ & \\
\hline $\begin{array}{l}\text { Digo que se ha construido el triángulo } \\
\mathrm{KZH} \text { con tres segmentos iguales a } \mathrm{A}, \mathrm{B} \text {, } \\
\mathrm{C} \text {. }\end{array}$ & \\
\hline
\end{tabular}

Figura 3. Enunciado de la Tarea 3 en ambas versiones del cuestionario. 
Por último, las fases de puesta en común fueron grabadas en video y transcritas para su posterior análisis. De este modo, mediante un proceso de triangulación de datos se obtuvo información en distintas fuentes (Flick, 2009) lo que, además de enriquecer el análisis, contribuye a mejorar la calidad de la investigación (Onwuegbuzie y Leech, 2007).

Las variables que se consideraron (sobre las que se centra principalmente nuestro análisis) y las fuentes de información relevantes para cada una de ellas son las siguientes:

- Seguimiento de las instrucciones por parte de los alumnos. Queríamos estudiar si los alumnos se ajustaban o no a las instrucciones proporcionadas en el enunciado de la tarea. Para determinarlo, comparamos estas instrucciones con los pasos seguidos por los alumnos en su construcción, observados tanto en el protocolo de construcción de GeoGebra como en las producciones escritas.

- Corrección de la construcción realizada en GeoGebra. Una construcción se considera correcta cuando su resultado final es susceptible de ser considerado una figura. Para determinarlo, realizamos ampliaciones en busca de posibles inexactitudes y recurrimos al test de arrastre para determinar las propiedades matemáticas que no se cumplen. En el caso de que detectáramos inexactitudes o que la construcción no pasara el test de arrastre, consideramos que estamos ante un dibujo y no ante una figura.

- Procedimiento empleado por los alumnos. Analizamos las herramientas de GeoGebra empleadas y el orden en que son utilizadas por los alumnos. Para determinarlo, recurrimos a las producciones escritas de los alumnos, al protocolo de construcción de GeoGebra (con atención especial a las prácticas de arrastre realizadas) y a las transcripciones de la fase de puesta en común.

Teniendo en cuenta los distintos objetivos, el carácter mixto de la investigación y la diferente naturaleza de los datos recogidos, utilizamos diversas herramientas de análisis. Para estudiar las variables 'corrección de la construcción' y 'seguimiento de las instrucciones' (que son de tipo categórico) así como la posible relación entre ellas y con la versión del cuestionario, se analizaron las producciones de los alumnos y sus ficheros GeoGebra. El análisis, de tipo cuantitativo y descriptivo, se realizó recurriendo al test $\chi^{2}$ de independencia (Blaikie, 2003) calculado mediante el software SPSS. Por otro lado, la variable 'procedimiento empleado con los alumnos' se estudió con un enfoque cualitativo e interpretativo 
a partir del análisis de contenido (Berg, 2007) de las producciones de los alumnos y de las transcripciones de sus intervenciones utilizando las herramientas analíticas señaladas anteriormente.

\section{RESULTADOS}

\subsection{ANÁLISIS CUANTITATIVO}

El primero de nuestros objetivos está relacionado con la dependencia entre las distintas variables consideradas y las distintas versiones del cuestionario. Para abordarlo desde un enfoque cuantitativo, la Tabla 2 recoge la información global relativa a las 51 producciones analizadas.

Tabla 2. Resultados globales

\begin{tabular}{|c|c|c|c|c|}
\cline { 2 - 5 } \multicolumn{1}{c|}{} & \multicolumn{2}{c|}{ Versión B } & \multicolumn{2}{c|}{ Versión C } \\
\cline { 2 - 5 } & $\begin{array}{c}\text { Construcción } \\
\text { correcta }\end{array}$ & $\begin{array}{c}\text { Construcción } \\
\text { incorrecta }\end{array}$ & $\begin{array}{c}\text { Construcción } \\
\text { correcta }\end{array}$ & $\begin{array}{c}\text { Construcción } \\
\text { incorrecta }\end{array}$ \\
\hline $\begin{array}{c}\text { Respetan } \\
\text { instrucciones }\end{array}$ & 9 & 5 & 10 & 4 \\
\hline $\begin{array}{c}\text { No respetan } \\
\text { instrucciones }\end{array}$ & 10 & 3 & 5 & 5 \\
\hline
\end{tabular}

Si realizamos los correspondientes test $\chi^{2}$ de independencia, se concluye que no existe relación entre ninguna de las parejas de variables consideradas a nivel global. Sin embargo, si consideramos una nueva variable, cuyos 6 valores vienen dados por el cruce de las tres tareas y las dos versiones, encontramos que existe relación (con un nivel de significatividad del 90\%) entre esta nueva variable y la corrección de la construcción realizada por los alumnos. En concreto, y tal y como se aprecia en la Figura 4, el peor rendimiento se ha dado en la versión C de la Tarea 1 y en la versión B de la Tarea 3. 


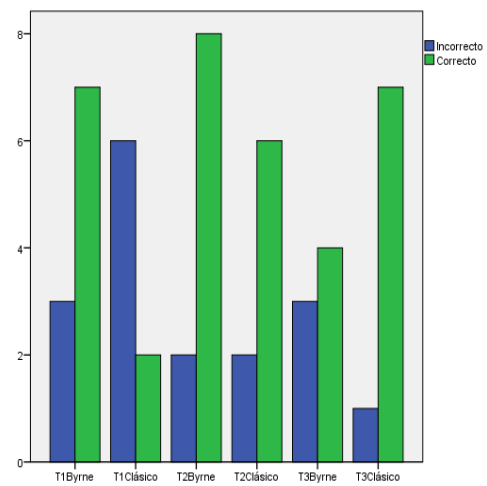

Figura 4. Corrección de las tareas en función de la versión del cuestionario.

A continuación, vamos a analizar más detalladamente cada una de las tareas de forma individual.

\subsubsection{Tarea 1}

En primer lugar, señalamos que sólo la mitad de los grupos participantes siguió al pie de la letra las instrucciones proporcionadas en el formulario al reproducir la construcción en GeoGebra. La sencillez de la tarea permitió que las construcciones realizadas por todos los grupos tuvieran como resultado (según la terminología presentada anteriormente) un dibujo de un cuadrado. Sin embargo, en la mitad de los grupos la construcción no dio lugar a una figura y su construcción se consideró incorrecta desde el punto de vista del test de arrastre.

Tabla 3. Resultados de la Tarea 1

\begin{tabular}{|c|c|c|c|c|}
\cline { 2 - 5 } \multicolumn{1}{c|}{} & \multicolumn{2}{c|}{ Versión B } & \multicolumn{2}{c|}{ Versión C } \\
\cline { 2 - 5 } & $\begin{array}{c}\text { Construcción } \\
\text { correcta }\end{array}$ & $\begin{array}{c}\text { Construcción } \\
\text { incorrecta }\end{array}$ & $\begin{array}{c}\text { Construcción } \\
\text { correcta }\end{array}$ & $\begin{array}{c}\text { Construcción } \\
\text { incorrecta }\end{array}$ \\
\hline $\begin{array}{c}\text { Respetan } \\
\text { instrucciones }\end{array}$ & 3 & 2 & 1 & 3 \\
\hline $\begin{array}{c}\text { No respetan } \\
\text { instrucciones }\end{array}$ & 4 & 1 & 1 & 3 \\
\hline
\end{tabular}


El seguimiento de las instrucciones se produjo con independencia de la versión del cuestionario con el que estaban trabajando los estudiantes. Del mismo modo, no hubo dependencia entre la corrección de la construcción y el seguimiento de las instrucciones (Tabla 3).

Sin embargo, como también se puede observar en la Tabla 3, los alumnos que completaron el modelo $B$ del cuestionario encontraron aparentemente menos dificultades y siete de ellos realizaron la construcción correctamente con GeoGebra. De hecho, si realizamos un test $\chi^{2}$ de independencia entre ambas variables (versión del cuestionario y corrección de la construcción), obtenemos que existe dependencia entre ellas con un nivel de confianza de aproximadamente el $90 \%$.

\subsubsection{Tarea 2}

Casi la mitad de los grupos participantes no siguió al pie de la letra las instrucciones proporcionadas en el formulario al reproducir la construcción en GeoGebra. Pese a que esta tarea era más compleja que la anterior, solo 4 de las 18 parejas realizaron una construcción incorrecta. No obstante, 3 de las construcciones incorrectas se pueden considerar un dibujo de la tangente pedida (pero no una figura). Un grupo no completó la construcción.

Tabla 4. Resultados de la Tarea 2

\begin{tabular}{|c|c|c|c|c|}
\cline { 2 - 5 } \multicolumn{1}{c|}{} & \multicolumn{2}{c|}{ Versión B } & \multicolumn{2}{c|}{ Versión C } \\
\cline { 2 - 5 } & $\begin{array}{c}\text { Construcción } \\
\text { correcta }\end{array}$ & $\begin{array}{c}\text { Construcción } \\
\text { incorrecta }\end{array}$ & $\begin{array}{c}\text { Construcción } \\
\text { correcta }\end{array}$ & $\begin{array}{c}\text { Construcción } \\
\text { incorrecta }\end{array}$ \\
\hline $\begin{array}{c}\text { Respetan } \\
\text { instrucciones }\end{array}$ & 5 & 0 & 5 & 1 \\
\hline $\begin{array}{c}\text { No respetan } \\
\text { instrucciones }\end{array}$ & 3 & 2 & 1 & 1 \\
\hline
\end{tabular}

Aunque la versión del cuestionario parece tener una clara influencia sobre el seguimiento de las instrucciones, esta no es estadísticamente significativa. Por su parte, no existe relación entre la versión del cuestionario y si la construcción es correcta o no. Sin embargo, si realizamos un test $\chi^{2}$ de independencia, observamos que sí existe dependencia estadística (al 90\%) entre el nivel de corrección 
y la construcción realizada y el respeto de las instrucciones. (Tabla 4). De hecho, los grupos que respetan las instrucciones obtienen mejores resultados que aquellos que no lo hacen.

\subsubsection{Tarea 3}

Tres grupos no llegaron a realizar esta tarea por falta de tiempo. De los restantes, prácticamente la mitad no respetaron las instrucciones. Tan solo 4 parejas que realizaron la tarea dieron como respuesta una construcción incorrecta (que no pasaba el test de arrastre), no llegando ni siquiera a construir un dibujo.

Tabla 5. Resultados de la Tarea 3

\begin{tabular}{|c|c|c|c|c|}
\cline { 2 - 5 } \multicolumn{1}{c|}{} & \multicolumn{2}{c|}{ Versión B } & \multicolumn{2}{c|}{ Versión C } \\
\cline { 2 - 5 } & $\begin{array}{c}\text { Construcción } \\
\text { correcta }\end{array}$ & $\begin{array}{c}\text { Construcción } \\
\text { incorrecta }\end{array}$ & $\begin{array}{c}\text { Construcción } \\
\text { correcta }\end{array}$ & $\begin{array}{c}\text { Construcción } \\
\text { incorrecta }\end{array}$ \\
\hline $\begin{array}{c}\text { Respetan } \\
\text { instrucciones }\end{array}$ & 1 & 3 & 4 & 0 \\
\hline $\begin{array}{c}\text { No respetan } \\
\text { instrucciones }\end{array}$ & 3 & 0 & 3 & 1 \\
\hline
\end{tabular}

En este caso, no existe ninguna relación entre la versión del cuestionario y el respeto de las instrucciones. Por su parte, aunque parece haber una influencia entre el respeto de las instrucciones y el nivel de corrección de la construcción, esta no resulta estadísticamente significativa. Finalmente, sí se aprecia relación de manera estadísticamente significativa (ver Tabla 5) entre la versión del cuestionario y si la construcción es correcta o no. De hecho, se puede observar que los alumnos que completaron el modelo B del cuestionario encontraron aparentemente más dificultades a la hora de realizar la construcción correctamente con GeoGebra.

\subsection{Análisis cualitativo}

El segundo de nuestros objetivos está relacionado con el análisis de las producciones realizadas por nuestros alumnos. Después de revisar dichas producciones emergen una serie de fenómenos que se muestran en la Tabla 6. En dicha tabla 
se presentan, de manera resumida, los indicadores de cada uno de los fenómenos y las herramientas que permiten su detección.

Tabla 6. Indicadores y herramientas para la detección de los distintos fenómenos identificados.

\begin{tabular}{|c|c|c|c|}
\hline & & & \\
\hline & \multirow[b]{2}{*}{ Indicadores } & \multicolumn{2}{|c|}{ Detectado mediante } \\
\hline & & Ampliación & $\begin{array}{l}\text { Test de } \\
\text { arrastre }\end{array}$ \\
\hline $\begin{array}{l}\text { Uso de medidas concretas } \\
\text { del dibujo }\end{array}$ & $\begin{array}{l}\text { Aparición de elementos geométri- } \\
\text { cos de medidas determinadas en el } \\
\text { proceso de construcción. }\end{array}$ & No & Sí \\
\hline $\begin{array}{l}\text { Suposición de propiedades } \\
\text { geométricas a partir de un } \\
\text { dibujo }\end{array}$ & $\begin{array}{l}\text { Aparición en el proceso de cons- } \\
\text { trucción de características no decla- } \\
\text { radas en el enunciado. }\end{array}$ & No & Parcialmente \\
\hline Arrastre guiado de objetos & $\begin{array}{l}\text { Aparición de objetos libres en pa- } \\
\text { sos intermedios del proceso de } \\
\text { construcción. }\end{array}$ & Sí & Sí \\
\hline Uso impropio 3 de GeoGebra & $\begin{array}{l}\text { Aparición de prácticas de "corta-pe- } \\
\text { ga" en el proceso de construcción. }\end{array}$ & Sí & Sí \\
\hline
\end{tabular}

A continuación, vamos a analizar detalladamente cada uno de estos cuatro fenómenos.

\subsubsection{Uso de medidas concretas del dibujo}

Algunos alumnos muestran, a la hora de realizar las construcciones, un cierto apego a las medidas concretas de los objetos geométricos construidos o proporcionados, por encima de las propiedades esenciales de estos objetos, que determinan los pasos del proceso de construcción solicitado. Esto se observa en la siguiente transcripción, relacionada con la Tarea 2 por ejemplo, donde se aprecia

\footnotetext{
${ }^{3}$ Entendemos por uso impropio del software la utilización de funcionalidades del programa de un modo no previsto por los diseñadores del mismo.
} 
la falta de discriminación entre propiedades esenciales (un punto es exterior a una circunferencia) y atributos específicos (un punto está a distancia cinco del centro):

Profesor: Y el radio ¿̇uánto habéis puesto?

Estudiante: Los valores que nos daban. Además, los valores también aparecían en aquél lado (en la ventana algebraica).

Otro posible ejemplo relacionado con este fenómeno se aprecia en la Tarea 3, donde algunos alumnos crearon nuevos segmentos con la herramienta 'segmento de longitud dada' a partir de las longitudes de los segmentos que se daban como datos. En la Figura 5 mostramos un momento intermedio de la construcción (tras el paso 14 del protocolo de construcción) donde podemos observar que los alumnos marcan las longitudes de los segmentos dados: en el paso 10 del protocolo de construcción marcan la longitud del segmento $A_{1} B_{1}$ (dado) y a continuación en el paso 14, marcan la longitud ED. Posteriormente repiten el mismo proceso con los segmentos $C_{1} D_{1}$ y $E_{1} F_{1}$.

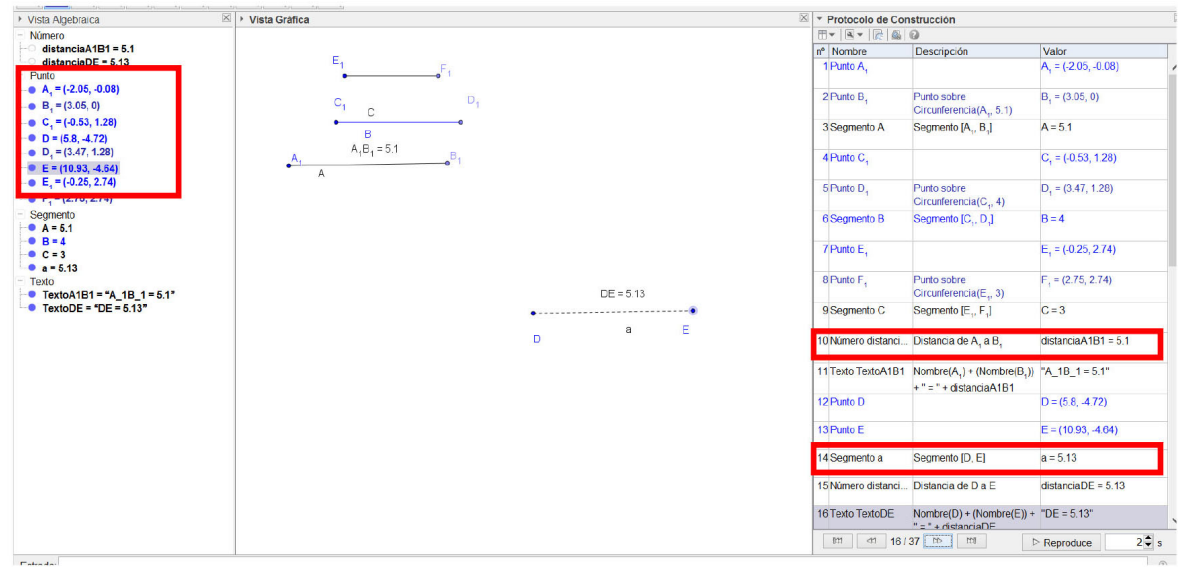

Figura 5. Ejemplo de uso de medidas concretas en la Tarea 3.

Estos alumnos no parecen asumir el carácter genérico de los tres segmentos dados, lo que podría estar relacionado con una cierta falta de comprensión del carácter general y no dependiente de las medidas concretas de un proceso de construcción geométrica. 


\subsubsection{Suposición de propiedades geométricas a partir de un dibujo}

Ciertas construcciones muestran, además de las propiedades geométricas requeridas, alguna otra propiedad extraída directamente de la ilustración que acompaña a las instrucciones. En el siguiente extracto (correspondiente a la explicación dada por los alumnos a la Figura 6 relacionada con la construcción de una perpendicular) observamos la extracción de información adicional (en este caso incorrecta) a partir de la ilustración que acompaña a las instrucciones:

Profesor: ¿Habéis seguido las instrucciones que se daban? ¿Habéis añadido alguna instrucción que no hubiera ahí? ¿Pensáis que habéis añadido algo?

Estudiante: [silencio] creo que hemos unido... bueno, no sé si ponía que uniéramos H con... no lo sé. Tampoco las hemos seguido a rajatabla, simplemente hemos empezado y luego ya hemos ido... siguiendo el dibujo.

$[\ldots]$

Profesor: ¿Y ha sido en ese momento cuando habéis pensado que había que trazar una perpendicular?

Estudiante: Sí, una perpendicular a $\mathrm{H}$.

Profesor: ¿Y eso viene en las instrucciones?

Estudiante: No

El comportamiento seguido por este grupo está muy relacionado con el intento de reproducir la ilustración, por encima del seguimiento de las instrucciones. En la Figura 6 (parte izquierda) vemos la ilustración proporcionada en el cuestionario. Pese a que lo parece, el segmento KH no es perpendicular al DE. Los alumnos, sin embargo, utilizaron la herramienta 'perpendicular' y, a partir de este paso, trataron de reproducir la ilustración dada tal y como se aprecia en la parte derecha de la citada figura.

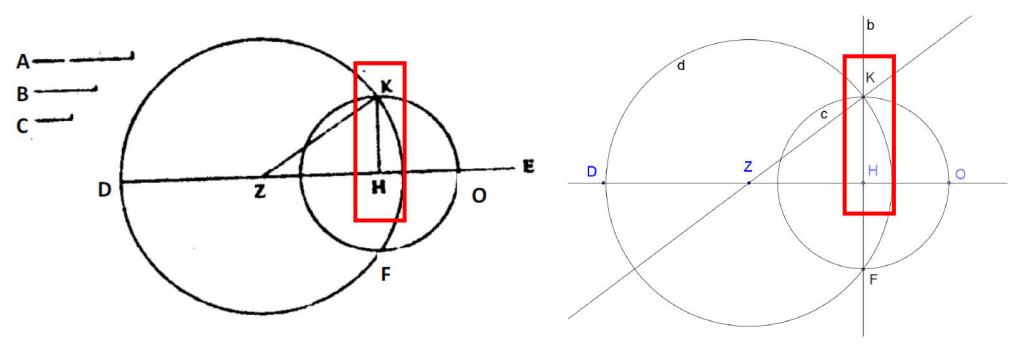

Figura 6. Imitación de la figura dada en la Tarea 3 
La ilustración que acompaña a las instrucciones es un dibujo, por tanto, no es posible extraer información teórica de la misma, sino que se le da un valor descriptivo. Esto forma parte del contrato didáctico establecido en nuestras clases de geometría. Si revisamos el protocolo de construcción de esta tarea (ver Figura 7), vemos el paso anterior a construir la perpendicular. Añadir la perpendicularidad facilita la construcción al dar a los alumnos un nuevo camino para encontrar la posición de un vértice como intersección de una circunferencia y una recta. No creemos que sea fruto de un despiste ya que no se ha pasado por alto un detalle o una instrucción, sino que se ha creado una nueva condición "siguiendo el dibujo" (en palabras de los estudiantes).

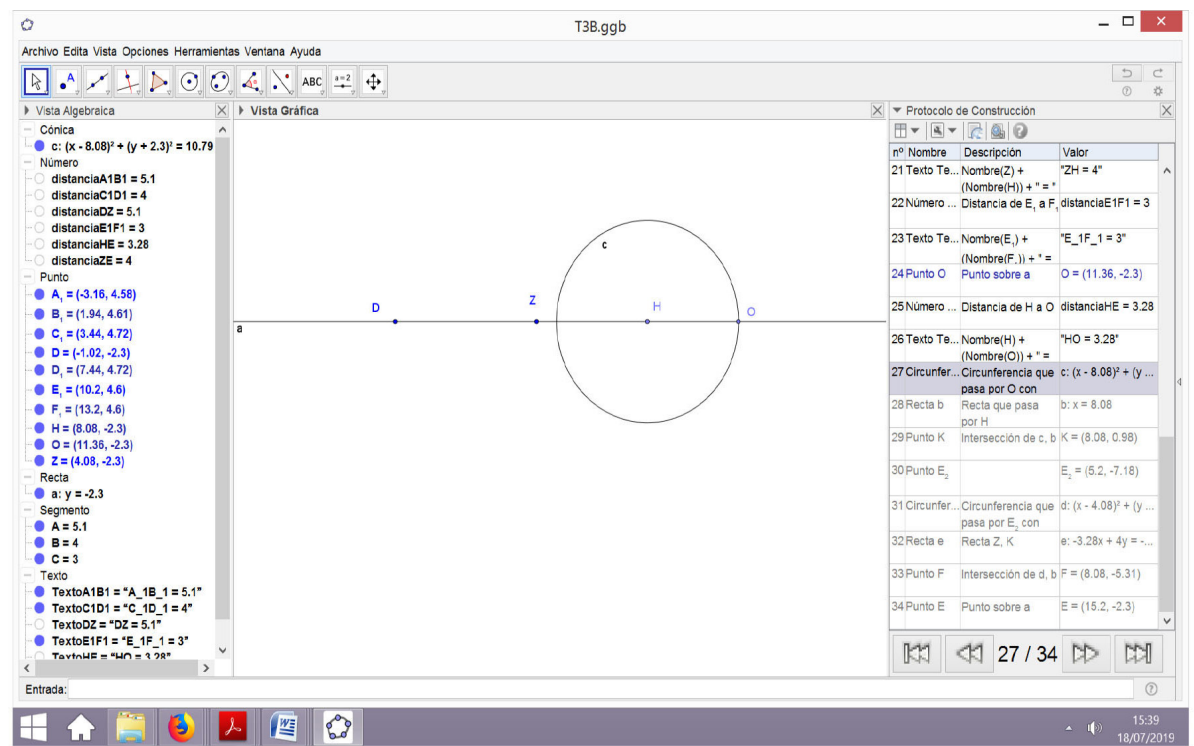

Figura 7. Imitación de la figura dada en la Tarea 3

\subsubsection{Arrastre guiado de objetos}

En algunas construcciones se observa, gracias a la revisión del protocolo de construcción, que, en lugar de trasladar a GeoGebra las propiedades matemáticas asociadas a las instrucciones proporcionadas, algunas parejas construyen objetos libres (que no dependen de los anteriores), para posteriormente ajustar 
la construcción y que se parezca lo más posible a la ilustración que acompaña a las instrucciones. En la transcripción siguiente, correspondiente a la tercera tarea, se observa este fenómeno.

Profesor: Vale, entonces, ¿cómo habéis conseguido C?

Estudiante: A ver, C, un punto, como no se especificaba, hemos cogido un punto cualquiera.

Profesor: ¿Y da la casualidad de que la tangente pasa por ahí?

Estudiante: Sí, bueno, lo hemos trampeado un poco, vale [risas] vale, pues al principio no nos salía y lo hemos movido y ya está.

Durante la construcción no se usa la propiedad matemática. En su lugar los alumnos construyen un objeto libre "hemos cogido un punto cualquierd" y tratan de ajustarlo posteriormente "al principio no nos salía y lo hemos movido y ya está".

Otro ejemplo de este fenómeno puede observarse en la Figura 8, correspondiente a la Tarea 2. Los alumnos ajustaron el radio de la circunferencia e haciendo que pasara por el punto $A$, además se arrastró el punto $D$ para que quedara aparentemente alineado con los puntos $E$ y $C$; se ayudaron para ello de una semirrecta que posteriormente ocultaron.

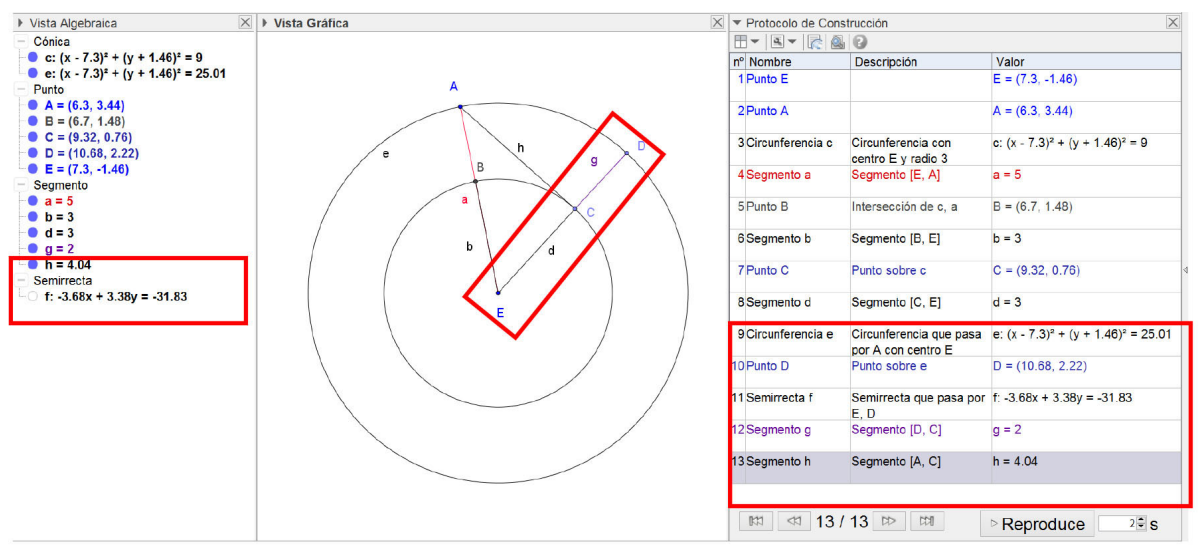

Figura 8. Ajuste de elementos a mano en la Tarea 2

Este fenómeno también es observable en la Tarea 1, realizando arrastres guiados de objetos que se construyeron sin haber impuesto todas las condiciones que 
los definen. Por ejemplo, para construir una perpendicular al segmento dado por uno de sus extremos, algunos grupos construyeron primero un punto arbitrario exterior al segmento, a continuación, trazaron una perpendicular al segmento por ese punto y luego la arrastraron hasta que visualmente pasara por el extremo del segmento (ver Figura 9).

Los alumnos no abordan la construcción de la perpendicular buscada como un proceso secuencial que consta de una serie de pasos ordenados jerárquicamente. En su lugar, construyen un objeto que cumple solo una de las propiedades requeridas (en este caso, la perpendicularidad) y realizan un arrastre guiado para tratar de que se cumplan las demás.

Paso 1

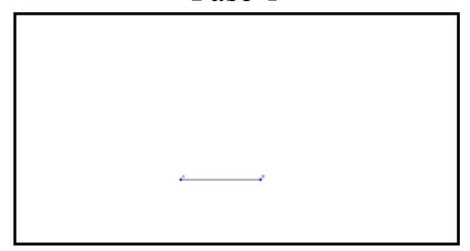

Paso 3

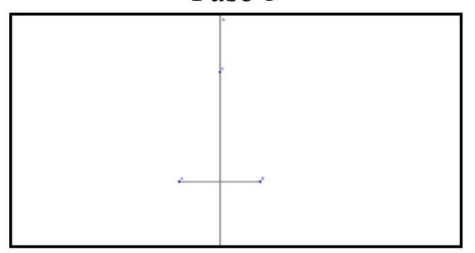

Paso 2

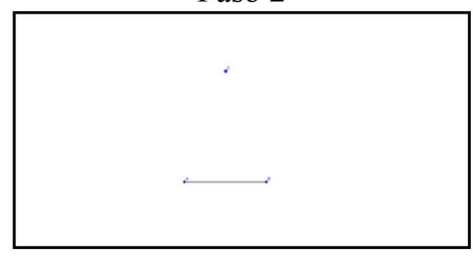

Paso 4

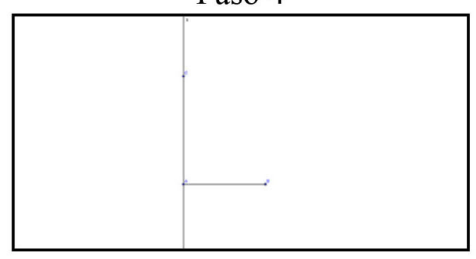

Figura 9. Arrastre guiado en la construcción de la perpendicular a una recta por un punto en la Tarea 1

\subsubsection{Uso impropio de GeoGebra}

En algunas ocasiones (ver la siguiente transcripción), los alumnos trasladan los elementos que se les proporcionan en el archivo de GeoGebra para utilizarlos en su construcción. En este proceso no logran hacer coincidir los elementos necesarios (en este caso los extremos de dos segmentos) para que la construcción cumpla las propiedades requeridas. 
Profesor: Cuando decís que habéis transportado...

Alumno: Bueno, hemos copiado el segmento...

Profesor: ¿Y con qué herramienta?

Alumno: Control C, control V.

Profesor: ¿Y para acercar y que estuviera donde queríais que estuviera?

Alumno: Con GGB si lo situabas encima, coincidía...

Como vemos, los alumnos han utilizado un elemento no matemático del software (la opción cortar y pegar). En GeoGebra, esta funcionalidad no está pensada para ser usada con objetos geométricos durante un procedimiento de construcción. En la Figura 10 se puede observar, haciendo una ampliación suficiente, que el proceso anterior es inadecuado ya que, tras trasladar el segmento, los extremos solo coinciden visualmente.

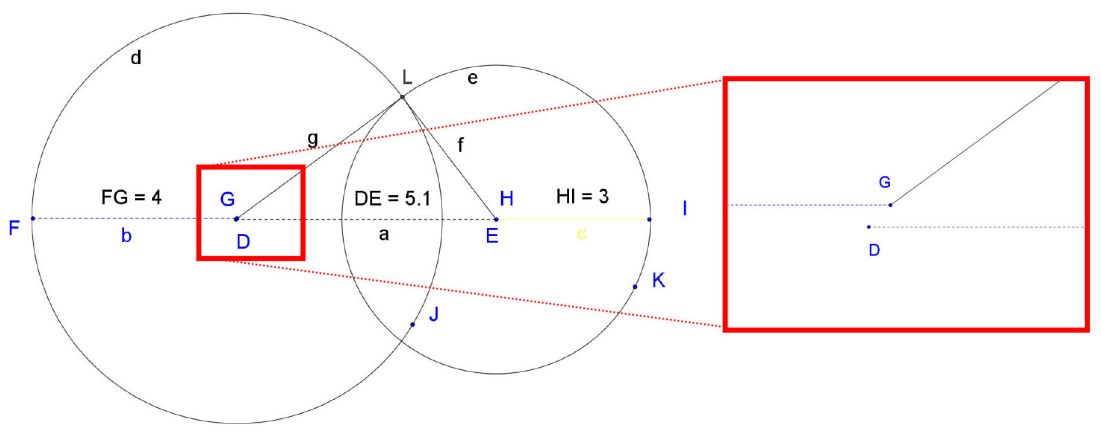

Figura 10. Traslación incorrecta de un segmento sobre otro en la Tarea 3

\section{DISCUSIÓN Y CONCLUSIONES}

Nuestro primer objetivo, consistía en analizar las posibles dependencias entre las variables consideradas (sistema de representación, seguimiento de instrucciones y nivel de corrección de la construcción). A este respecto, al analizar globalmente las tareas no se ha encontrado ninguna relación estadísticamente significativa entre las variables consideradas. Sin embargo, al considerar como variable el par (tarea, sistema de representación) sí se ha logrado detectar una relación significativa entre esta nueva variable y el nivel de corrección de la construcción. Esto pone de manifiesto la importancia de las tareas a la hora de 
comprender la influencia del sistema de representación sobre la capacidad de los alumnos para llevar a cabo la construcción correctamente. Es decir, parece que el uso de un sistema de representación u otro es más o menos adecuado no en términos absolutos, sino en función de la tarea específica en la que se utiliza. En cualquier caso, los alumnos de Magisterio que realizaron construcciones a partir de la edición de Byrne tuvieron una tasa de éxito algo mayor (70\%) que los que lo intentaron a partir de la versión clásica de la editorial Gredos (62\%).

Es interesante señalar que, entre aquellos grupos que no respetaron las instrucciones, hay un porcentaje mucho mayor de acierto en la construcción en la versión B (un 77\%) que en la versión C (un 50\%). Por su parte, entre aquellos alumnos que respetaron las instrucciones rigurosamente, la versión $\mathrm{C}$ proporciona un porcentaje de aciertos ligeramente mayor (71\% frente a $64 \%$ ). Así pues, el sistema de representación de Byrne parece penalizar en menor medida el que los alumnos sigan un camino propio mientras que, al ser las instrucciones proporcionadas en la versión clásica más rigurosas y completas, su seguimiento favorece el éxito en la tarea.

Una posible explicación al fenómeno anterior puede quizás encontrarse en la distinta naturaleza de la ilustración que acompaña a las proposiciones en cada caso (Mesquita, 1998). Por un lado, puede considerarse como un elemento más de las instrucciones, a veces resulta indispensable para poder seguirlas y completar la construcción. Por otro lado, también puede verse como un elemento de comprobación de la construcción que un lector pudiera hacer. En la puesta en común se puso de manifiesto cómo los alumnos utilizan la utilizan en estos dos sentidos. En la transcripción siguiente se muestra como uno de los grupos utiliza esta ilustración de una u otra forma según la tarea:

Profesor: ¿Os ha servido de algo la figura? La que os dan...

Alumno: Sí, para hacernos una idea de cómo debía ser el resultado final. Ya sabíamos la respuesta. En la otra nos ha servido más, si no hubiera sido por el dibujo no la hubiéramos sacado.

La expresión "para hacernos una idea de cómo debía ser el resultado final" pone de manifiesto el uso como elemento de comprobación, mientras que la expresión "si no hubiera sido por el dibujo no lo hubiéramos sacado" apunta al uso de información contenida en la ilustración.

No obstante, creemos que la ilustración que acompaña a la versión de Byrne tiene principalmente un carácter de figura, de la que puede extraerse información 
y que juega un papel de complemento a las instrucciones (lo que se pone de manifiesto, por ejemplo, en la omisión de pasos en la Tarea 2 o de indicaciones precisas de la ubicación de elementos de la construcción en la Tarea 1) mientras que en la versión clásica se trata principalmente de un dibujo. Esta distinta naturaleza (Mesquita, 1998) quizás también puede explicar en parte que el porcentaje de grupos que no siguieron las instrucciones fuera ligeramente mayor en la versión B (sobre un 48\%) que en la versión C (sobre un 41\%).

Los fenómenos identificados se reparten de manera desigual en las tareas estudiadas. El fenómeno 1 (uso de medidas concretas) se ha detectado únicamente en la Tarea 2. El fenómeno 2 (suposición de propiedades geométricas a partir del dibujo) aparece en las Tareas 2 y 3, pero no en la Tarea 1. Puesto que los alumnos recurren a la ilustración que acompaña a las instrucciones especialmente en aquellas construcciones con un mayor grado de dificultad, no era esperable la aparición de este fenómeno en la construcción del cuadrado. El fenómeno 3 (arrastre guiado de objetos) es el más común y aparece en las todas las tareas. Esto puede estar relacionado con un escaso conocimiento del manejo de GeoGebra y de ahí su transversalidad. Finalmente, el fenómeno 4 (uso impropio de GeoGebra) ha aparecido únicamente en la Tarea 3 y está asociado específicamente a la acción de trasladar un segmento dado a otro lugar.

Aunque hemos tratado de caracterizar estos fenómenos, no pensamos que sean completamente independientes unos de otros, sino que están relacionados y en ocasiones se superponen unos y otros. De hecho, los fenómenos 1 y 2 tienen una mayor componente matemática mientras que los fenómenos 3 y 4 están más relacionados con los procesos de instrumentalización e instrumentación asociados al uso de GeoGebra. Estos dos factores fueron identificados por Ruiz-López (2017) como obstáculos en el proceso de génesis instrumental usando GeoGebra. En la Tabla 7, presentamos un resumen de la información proporcionada por cada uno de estos fenómenos y su relación con elementos teóricos de la literatura. 
Tabla 7. Información proporcionada y relación con elementos teóricos de los distintos fenómenos identificados.

\begin{tabular}{|c|c|c|}
\hline & \multirow{2}{*}{ Información } & \multirow{2}{*}{ Relacionado con } \\
\hline & & \\
\hline $\begin{array}{l}\text { Uso de medidas } \\
\text { concretas del } \\
\text { dibujo }\end{array}$ & $\begin{array}{l}\text { El estudiante no ve la construcción como } \\
\text { un proceso general o no es capaz de lle- } \\
\text { varlo a cabo. } \\
\text { El estudiante no discrimina las propieda- } \\
\text { des esenciales y las accesorias de la ilus- } \\
\text { tración que acompaña las instrucciones. }\end{array}$ & $\begin{array}{l}\text { Posibles deficiencias en el co- } \\
\text { nocimiento matemático. } \\
\text { Confusión entre dibujo y figura }\end{array}$ \\
\hline $\begin{array}{l}\text { Suposición de } \\
\text { propiedades } \\
\text { geométricas a } \\
\text { partir de un dibujo }\end{array}$ & $\begin{array}{l}\text { El estudiante no asume el papel de dibujo } \\
\text { de la ilustración que acompaña las instruc- } \\
\text { ciones (ruptura del contrato didáctico). }\end{array}$ & Confusión entre dibujo y figura. \\
\hline $\begin{array}{l}\text { Arrastre guiado de } \\
\text { objetos }\end{array}$ & $\begin{array}{l}\text { El estudiante no traslada las propiedades } \\
\text { matemáticas a GeoGebra, sino que trata de } \\
\text { aproximar la apariencia del resultado a la } \\
\text { ilustración que acompaña las instrucciones. } \\
\text { El estudiante no asume la naturaleza se- } \\
\text { cuencial de la construcción. }\end{array}$ & $\begin{array}{l}\text { Posibles deficiencias en el co- } \\
\text { nocimiento matemático }\end{array}$ \\
\hline $\begin{array}{l}\text { Uso impropio de } \\
\text { GeoGebra }\end{array}$ & $\begin{array}{l}\text { El estudiante utiliza elementos no matemá- } \\
\text { ticos de GeoGebra con un sentido mate- } \\
\text { mático impropio. }\end{array}$ & $\begin{array}{l}\text { Bajo nivel de instrumentali- } \\
\text { zación }\end{array}$ \\
\hline
\end{tabular}

Si concretamos más, los fenómenos 1 y 2 provienen principalmente de trabajar de forma concreta con los datos proporcionados, ya sea extrayendo información de tipo métrico (fenómeno 1) o suponiendo propiedades a partir de la ilustración que se les proporcionaba (fenómeno 2). En el fenómeno 1, la construcción realizada por los alumnos da lugar a un dibujo, pero no a una figura y sería únicamente detectable mediante la realización de un test de arrastre. Por su parte, en el fenómeno 2, la construcción puede no dar siquiera lugar a un dibujo en función de si la propiedad inferida es compatible o no con la construcción solicitada, poniéndose de manifiesto las inexactitudes inherentes al dibujo (Parzysz, 1988). En la Tarea 3, por ejemplo, los lados proporcionados como datos no permiten la construcción de un triángulo rectángulo y de ahí el fenómeno detectado en la Figura 6. En el caso hipotético de que hubiera sido 
posible la introducción de una restricción adicional (ser rectángulo, por ejemplo) se habría dado lugar a un dibujo pero no a una figura, pues al cambiar la longitud de los lados la restricción dejaría de ser compatible con los datos. En esta situación, el test de arrastre mostraría que la solución es, como mucho, parcialmente correcta ya que la construcción podría cumplir las condiciones requeridas y además las impuestas por la ilustración que las acompaña, lo que daría un subconjunto de las soluciones. No obstante, sería necesario reflexionar sobre las limitaciones de los objetos que aparecerían al realizar dicho test. Además, en esta situación se pone de manifiesto una cierta falta de comprensión de la jerarquía de la información gráfica y de su subordinación a las instrucciones que acompaña (Talmon y Yerushalmy, 2004). A este respecto, autores como Capponi y Laborde (1991, p. 220) ya señalan que "...la variabilidad de elementos de una figura no puede ser explicada por un dibujo. En particular, el campo de variación de estos elementos tampoco está explicitado en el dibujo".

Por su parte, consideramos que los fenómenos 3 y 4 podrían estar relacionados en siguiente sentido: los ajustes que se realizan a mano vienen permitidos por el entorno tecnológico, es decir no se pueden realizar cuando se realiza la construcción con lápiz y papel. Se estaría dando validez matemática a aquello que el programa es capaz de hacer. En ambos casos podría haber parejas que hacen ajustes porque el programa lo permite, sin plantearse la validez matemática una vez que es posible (y por tanto válido) tecnológicamente. En ambos casos ni siquiera se obtienen dibujos; podríamos decir que en ambos fenómenos se da un cierto intento de suplir la herramienta "limitar-liberar objeto" para conseguir que un determinado objeto adquiera las relaciones de incidencia deseadas.

En el fenómeno 3, los alumnos utilizan algunas de las herramientas de GeoGebra (sobre todo para la construcción de objetos) y el ajuste se realiza sobre el producto de las mismas (sobre todo para asegurar las relaciones de incidencia entre los objetos construidos). Este modo de actuar muestra un nivel bajo de instrumentación (Iranzo y Fortuny, 2009) e ilustra una falta de comprensión de la naturaleza secuencial del proceso de construcción en el que un paso se sustenta en los anteriores y sustentará a los siguientes (Talmon y Yerushalmy, 2004). Este fenómeno sería detectable mediante la realización de un test de arrastre y una ampliación. En el fenómeno 4, por su parte, ni siquiera se utilizan herramientas matemáticas específicas de GeoGebra para construir objetos (se mueven o copian objetos dados). En este fenómeno se observa una incorrecta 
instrumentalización del entorno tecnológico que les resuelve lagunas del ámbito matemático. Esta colisión podría ser resuelta a veces por un adecuado uso de la herramienta de GeoGebra "limitar-liberar objeto" que permite en ocasiones que un objeto se "adhiera" a otro con lo que, de alguna forma, adquiriría las propiedades matemáticas del objeto receptor. Esta situación pone de manifiesto la necesidad de combinar adecuadamente los conocimientos matemáticos con los tecnológicos para que el instrumento tecnológico medie de forma positiva en la adquisición de los conceptos geométricos (Hollebrands, 2007). Este fenómeno es más fácilmente detectable mediante el uso de la herramienta zoom para realizar una ampliación, que muestra que los alumnos ni siquiera realizan un dibujo correcto. No obstante, también sería posible poner en evidencia este fenómeno mediante el test de arrastre.

Como venimos señalando, y tal como hemos visto en la Tabla 6, los fenómenos identificados varían según la herramienta que nos permite detectarlos. Aunque el test de arrastre es una herramienta potente que permite detectar la incorrección de la construcción en todos los casos, el uso de ampliaciones permite distinguir aquellos fenómenos que están relacionados más específicamente con el uso de GeoGebra. Así pues, en función del ámbito en que se esté desarrollando la actividad, de los destinatarios y objetivos de la misma 0 tipo de fenómeno detectado por el docente, éste puede elegir entre recurrir a una u otra herramienta.

Finalmente, teniendo en cuenta las anteriores consideraciones, podría ser interesante llevar a cabo una investigación encaminada a determinar criterios que nos permitan elegir uno u otro sistema de representación en función de la tarea que se esté llevando a cabo. A este respecto, aunque a primera vista el sistema de representación utilizado en la obra de Byrne parece más accesible (y, de hecho, en dos de las tres tareas ha dado resultados algo mejores), en algunos casos conlleva una sobreutilización de la ilustración que acompaña a las instrucciones que, como hemos visto en el caso de los fenómenos 1 y 2, puede suponer un cierto obstáculo. Otra posible línea de investigación podría encaminarse a desarrollar un sistema de representación "híbrido" en el que se tomaran aquellos elementos que se consideren más ventajosos de cada uno de los sistemas de representación que hemos manejado aquí. 


\section{AGRADECIMIENTOS}

Trabajo financiado parcialmente por el Gobierno de Aragón y el Fondo Social Europeo (Grupo "Investigación en Educación Matemática") y por el Ministerio de Economía y Competitividad de España (Proyecto EDU2015-65378-P).

\section{REFERENCIAS}

Arnal-Bailera, A. y Oller-Marcén, A.M. (2017). Formación del profesorado y demostración matemática. Estudio exploratorio e implicaciones. Boletim de Educaçao Matemática, 31(57), 135-157.

Arzarello, F., Olivero, F., Paola, D. y Robutti, O. (2002). A cognitive analysis of dragging practises in Cabri environments, Zentralblatt fur Didaktik der Mathematik, 34(5), 66-72.

Berg, B.L. (2007). Qualitative research methods for the social sciences. Allyn and Bacon. Blaikie, N. (2003). Analyzing Quantitative Data. SAGE.

Byrne, O. (1847). The first six books of the elements of Euclid in which coloured diagrams and symbols are used instead of letters for the greater ease of learners. William Pickering.

Castro, E. y Castro, E. (1997). Representaciones y modelización. En L. Rico (Coord.) La Educación Matemática en la Enseñanza Secundaria (pp. 95-124). Horsori-ICE

Capponi, B., y Laborde, C. (1991). Cabri-géomètre, un environnement pour l'apprentissage de la géométrie élémentaire. Actes de la Vlème école d'été de didactique des mathématiques et de l'informatique, Plestin les grèves, 220-222.

Drijvers, P. (2012). Teachers transforming resources into orchestrations. En G. Gueudet, B. Pepin y L. Trouche (Eds.), From text to 'lived' resources: mathematics curriculum materials and teacher development (pp. 265-281). Springer.

Duval, R. (2004). A crucial issue in mathematics education: The ability to change representation register. En M. Niss (Ed.) Proceedings of the Tenth International Congress on Mathematical Education (pp. 1-17). IMFUFA.

Duval, R. (2006). A cognitive analysis of problems of comprehension in a learning of mathematics. Educational Studies in Mathematics, 61, 101-131.

Elliot, R. y Timulak, L. (2005). Descriptive and interpretive approaches to qualitative research. En Miles, J. y Gilbert, P. (Eds.) A handbook of research methods for clinical and health psychology (pp. 147-159). Oxford University Press.

Euclides (Trd. en 1994). Elementos.Libros I-IV. Madrid, España: Gredos.

Flick, U. (2009). An Introduction to Qualitative Research. SAGE. 
Hernández, R., Fernández, C, y Baptista, P. (2010). Metodología de la Investigación. McGraw Hill.

Gómez, P. (2002). Análisis didáctico y diseño curricular en matemáticas. Revista EMA, 7(3), 251-292.

Guin, D. y Trouche, L. (2002). Mastering by the teacher of the instrumental genesis in CAS environments: necessity of intrumental orchestrations. Zentralblatt für Didaktik der Mathematik, 34(5), 204-211.

Gutiérrez, A. (2005). Aspectos metodológicos de la investigación sobre aprendizaje de la demostración mediante exploraciones con software de geometría dinámica. En A. Maz, B. Gómez y M. Torralba (Eds.), Actas del IX Simposio de la Sociedad Española de Investigación en Educación Matemática (SEIEM) (pp. 27-44). Córdoba, España: Universidad de Córdoba.

Hollebrands, K. F. (2007). The role of a dynamic software program for geometry in the strategies high school mathematics students employ. Journal for research in mathematics education, 38(2), 164-192.

Iranzo, N. y Fortuny, J.M. (2009). La influencia conjunta del uso de GeoGebra y lápiz y papel en la adquisición de competencias del alumnado. Enseñanza de las Ciencias, 27(3), 433-446.

Laborde, C.; Capponi, B. (1994). Cabri-Géomètre constituant d'un milieu pour l'apprentissage de la notion de figure géométrique, Recherches en Didactique des Mathématiques,14.1/2, 165-209.

Mariotti, M.A. y Bartolini, M.G. (1998). From drawing to construction: teacher's mediation within the Cabri environment. En K. Newstead y A. Olivier (Eds.). Proceedings of the 22nd Conference of the International Group for the Psychology of Mathematics Education (pp. 180-195). Stellenbosch: PME.

Marmolejo, G. A., y Vega, M. B. (2012). La visualización en las figuras geométricas: Importancia y complejidad de su aprendizaje. Educación matemática, 24(3), 7-32.

Mesquita, A. L. (1998). On conceptual obstacles linked with external representation in geometry. The Journal of Mathematical Behavior, 17(2), 183-195.

McGee, D. y Moore-Russo, D. (2015). Using a Technology-Supported Approach to Preservice Teachers' Mathematical Fluency: Unifying Mathematical Concepts and their Representations. Contemporary Issues in Technology and Teacher Education, 15(4), 489-513.

Onwuegbuzie, A.J., y Leech, N.L. (2007). Validity and qualitative research: an oxymoron? Quality y Quantity, 41(2), 233-249.

Özerem, A. (2012). Misconceptions in Geometry and suggested solutions for seventh grade students. Procedia-Social and Behavioral Sciences, 55, 720-729. 
Parzysz, B. (1988). "Knowing" vs "seeing". Problems of the plane representation of space geometry figures. Educational Studies in Mathematics, 19(1), 79-92.

Pole, K. (2009). Diseño de metodologías mixtas. Una revisión de las estrategias para combinar metodologías cuantitativas y cualitativas. Renglones, revista arbitrada en ciencias sociales y humanidades, 60, 37-42.

Rabardel, P. (1995). Les hommes et les technologies, approche cognitive des instruments contemporains. París, Francia: Armand Colin.

Rezat, S., y Sträßer, R. (2012). From the didactical triangle to the socio-didactical tetrahedron: artifacts as fundamental constituents of the didactical situation. Zentralblatt für Didaktik der Mathematik, 44(5), 641-651.

Rico, L. (2013). El método del análisis didáctico. Unión. Revista Iberoamérica de Educación Matemática, 33, 11-27.

Sandoval, I.T. (2009). La geometría dinámica como una herramienta de mediación entre el conocimiento perceptivo y el geométrico. Educación Matemática, 21(1), 5-27.

Ruiz-López, N. (2018). The instrumental genesis process in future primary teachers using Dynamic Geometry Software. International Journal of Mathematical Education in Science and Technology, 49(4), 481-500.

Talmon, V., y M. Yerushalmy (2004). Understanding dynamic behaviour: Parent-child relations in dynamic geometry environments, Educational Studies in Mathematics, 57(1), pp. 91-119.

Teddlie, C., y Tashakkori, A. (2009). Foundations of mixed methods research: Integrating quantitative and qualitative approaches in the social and behavioral sciences. London: SAGE.

Trouche, L. (2004). Managing the complexity of human/machine interactions in computerized learning environments: guiding students' command process through instrumental orchestrations. International Journal of Computers for Mathematical Learning, 9(3), 281-307.

ANTONIO M. OLLER-MARCÉN

Dirección: Centro Universitario de la Defensa de Zaragoza Ctra. de Huesca s/n, 50090, Zaragoza (España)

Teléfono: $\quad+34976739611$ 